\title{
Die Arbeitsgruppe „Aus- und Fortbildung“ der deutschen Sektion der AO-International
}

\author{
K. H. Winker
}

\section{Zusammenfassung}

Die Arbeitsgruppe „Aus- und Fortbildung" der deutschen Sektion der internationalen Arbeitsgemeinschaft für Osteosynthesefragen (AO) koordiniert und unterstützt wissenschaftliche Fortbildungsveranstaltungen, organisiert und strukturiert AO-Kurse für Ärzte und OP-Personal. Damit wird die Diskussion und die Verbreitung der AO-Prinzipien zur Analyse und Behandlung von Verletzungen und Erkrankungen des Bewegungsapparates möglich.

Nach Gründung der Arbeitsgemeinschaft für Osteosynthesefragen (AO) 1958 begannen kurz danach (1960) die ersten Schulungsaktitvitäten, die weltbekannten AO-Kurse für Ärzte in Davos. Wenig später (1963) wurden die ersten OP-Schwestern in den AO-Techniken ausgebildet.

Neben Forschung, Entwicklung und Qualitätssicherung ist der Bereich Schulung (Education) der wichtigste Grundpfeiler der AO-Philosophie. Heute existiert ein weltweites Netz an AO-Schulungsaktivitäten: bis Ende 1999 wurden in mehr als 70 Ländern mehr als 120000 Ärzte und mehr als 5500 OP-Schwestern ausgebildet. Das Kurssystem der AO ist national und international beispielhaft, oft kopiert, selten erreicht. Kaum ein Mediziner, der heute nicht während seines Studiums die Behandlung von Frakturen nach den Richtlinien der AO lernt, kaum ein Arzt, der nicht während seiner chirurgischen Weiterbildung einen AO-
Kurs besucht. In der Schulmedizin sind die Prinzipien der AO in den vergangenen 40 Jahren national und international zum Standard in der operativen Knochenbruchbehandlung geworden.

Sich auf diesem Erfolg auszuruhen würde Rückschritt bedeuten. Gutes zu verbessern, Kursformate zeitgemäß zu gestalten, heißt weiterhin interessant und attraktiv bleiben.

Folgerichtig wurde 1995 durch die deutsche Sektion der AO-International die „Arbeitsgruppe Aus- und Fortbildung“" ins Leben gerufen mit genau dieser Aufgabenstellung: Kurse und andere Veranstaltungsformate didaktisch, inhaltlich und strukturell zu überdenken und neue Erkenntnisse sowie technische Entwicklungen in das bewährte System mit aufzunehmen:

- der AO-Kurs Trauma I wird seit 1999 nach einem modularen Konzept durchgeführt, zusammengefasst dargestellt in einem Leitfaden für Referenten und Instruktoren

a der AO-Kurs Trauma II schulte im Oktober 2000 weltweit erstmals die Technik der OP-Navigation.

- Eine eigene Veranstaltung „teaching the teachers" vermittelte im Frühjahr 1999 Referenten und Instruktoren von AO-Kursen professionelles didaktisches Wissen, 2001 wird eine Folgeveranstaltung stattfinden.

- Praktische Übungen, Diskussionen und interaktives Lernen nehmen einen immer größeren Raum bei allen AO-Kursen ein.

- Die Erstellung von Präsentationen mit elektronischen Medien (power point etc.) war Inhalt einer Fortbildungsveranstaltung im September 2000.

Die Arbeitsgruppe hat für die kommenden Jahre viel vor: So soll die Struktur
AO-Kurs Trauma I

Prinzipien der operativen Knochenbruchbehandlung

\section{AO-Kurs Trauma II}

Fortschritte und Probleme in der operativen Knochenbruchbehandlung (erstmals Oktober 2000)

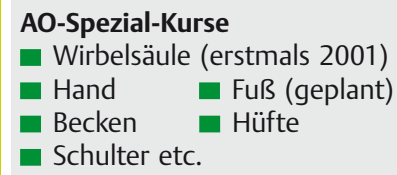

Spezielle Themen zu Instrumenten, Implantaten, neuen Techniken oder Technologien (LISS, Marknagelsysteme, LCP etc.)

AO-Kurs für OP-Personal AO-Kurs für leitendes OP-Personal

\section{AO-Seminar/Symposium}

Wissenschaftliche und praktische Bearbeitung spezieller Themenkomplexe nach Körperregionen, Verletzungen oder Behandlungsprinzipien

Systematik der Veranstaltungen der deutschen Sektion der AO-International

der AO-Kurse für OP-Personal überarbeitet, AO-Wirbelsäulen-Kurse etabliert und Schulungsmodelle für die Endoprothetik in der Unfallchirurgie erarbeitet werden. Natürlich wird der innovative Bereich der computerunterstützten Chirurgie viel Raum einnehmen. Also, packen wir's an!

Prof. Dr. med. K. H. Winker Leiter der AG Aus- und Fortbildung

Klinik für Unfallchirurgie Klinikum Erfurt

Nordhäuser Straße 74 99089 Erfurt 